\title{
Pichia guilliermondii
}

National Cancer Institute

\section{Source}

National Cancer Institute. Pichia guilliermondii. NCI Thesaurus. Code C77172.

A species of yeast in the phylum Ascomycota whose asexual or anamorphic form is known as Candida guilliermondii. 